\title{
ON PUISEUX SERIES \\ WHOSE CURVES PASS THROUGH AN INFINITY OF ALGEBRAIC LATTICE POINTS
}

\author{
BY DAVID LEE HILLIKER AND E. G. STRAUS ${ }^{1}$
}

1. Introduction. Runge [4] proved that certain binary Diophantine equations have only finitely many solutions. Here we give an argument concerning lattice points represented by Puiseux series which proves Runge's Theorem and permits a generalization which shows that there are only finitely many solutions in integers - subject to suitable restrictions-of an algebraic number field. As in the case of Runge's Theorem upper bounds for the absolute value of each solution can be computed, by the methods of the proof.

Let

$$
F(x, y)=\sum_{i=0}^{d_{1}} \sum_{j=0}^{d_{2}} a_{i j} x^{i} y^{j} \in \mathbf{C}[x, y]
$$

be of degree $d_{1}$ and $d_{2}$ in $x$ and $y$, respectively. Let $\lambda>0$. We define the $\lambda$-leading part, $F_{\lambda}(x, y)$, of $F(x, y)$ to be the polynomial consisting of the sum of all terms $a_{i j} x^{i} y^{j}$ of $F(x, y)$ for which $i+\lambda j$ is maximal, for that fixed value of $\lambda$. We define the leading part, $\tilde{F}(x, y)$, of $F(x, y)$ to be the sum of all such terms as $\lambda$ varies.

We say that an irreducible polynomial

$$
F(x, y) \in \mathbf{Z}[x, y]
$$

satisfies Runge's Condition unless there exists a $\lambda$ so that $\tilde{F}=F_{\lambda}$ is a constant multiple of a power of an irreducible polynomial.

Runge's Theorem can now be conveniently formulated: If $F(x, y)$ satisfies Runge's Condition, then the Diophantine equation $F(x, y)=0$ has only finitely many solutions $(x, y) \in \mathbf{Z}^{2}$.

Let $L$ denote an algebraic number field of degree $t$. Let the conjugates of $\theta \in L$ be denoted by $\theta^{(1)}=\theta, \theta^{(2)}, \theta^{(3)}, \ldots, \theta^{(t)}$, and let

$$
|\theta|=\max _{1 \leq \tau \leq t}\left|\theta^{(\tau)}\right| \text {. }
$$

Denote the ring of algebraic integers in $L$ by $O_{L}$. We say that $(x, y) \in O_{L}^{2}$ is an $L$-lattice point. We consider certain analytic functions $y=f(x)$, of a complex

Received by the editors February 3, 1982 and, in revised form, August 13, 1982.

1980 Mathematics Subject Classification. Primary 14H05; Secondary 10B10, 10B15.

Key words and phrases. Algebraic function, Diophantine equation, Puiseux series.

${ }^{1}$ Work of the second author was supported in part by NSF Grant MCS 79-03162. 
variable $x$, represented by Puiseux series

$$
f(x)=\sum_{n=-m}^{\infty} a_{n} x^{-n / e},
$$

$m, e \in \mathbf{Z}, a_{-m} \neq 0, e>0$, in a neighborhood of infinity, $|x|>R$.

THEOREM 1. Let $y=f(x)$ where the Puiseux coefficients $a_{n}$, for $n \leq N$, lie in an algebraic number field $K$ of degree $s$. Let $M$ be the number of pairs of integers $(\mu, \nu)$ with

$$
1 \leq \mu \leq \nu m / e, \quad 1 \leq \nu<s e .
$$

Consider L-lattice points $(x, y)$ that satisfy

(i) $y^{\left(\tau_{0}\right)}=f\left(x^{\left(\tau_{0}\right)}\right)$, for some $\tau_{0}$ with $\left|x^{\tau_{0}}\right| \geq|x|^{c_{1}}$,

(ii) $|y| \leq c_{2}|x|^{c_{3}}$,

(iii) $|1 / x| \leq c_{4}|x|^{c_{5}}$,

where $c_{1}, c_{2}, c_{3}, c_{4}$, and $c_{5}$ are positive constants with

$$
c_{5}<c_{1} / e s(t-1) \text {. }
$$

Suppose that

$$
\begin{aligned}
& N \geq M e+m(s e-1) /\left(c_{1}-c_{5} e s(t-1)\right), \\
& N \geq c_{3} e / c_{5}, \quad N \geq m / c_{5} .
\end{aligned}
$$

Then all these L-lattice points $(x, y)$ satisfy an equation $P(x, y)=0$ where $P(x, y) \in \mathbf{Z}[x, y], \operatorname{deg}_{y} P \leq$ se, and $P_{\lambda}$ is a monomial for all $\lambda \neq m / e$. For $\lambda=$ $m / e, P_{\lambda}$ is a constant multiple of a power of $x$ times a power of an irreducible polynomial in $\mathbf{Z}[x, y]$. Moreover, $\tilde{P}=P_{m / e}$. If there is an infinity of L-lattice points satisfying (i), (ii) and (iii), then $P(x, f(x)) \equiv 0$.

We deduce, in [3], Runge's Theorem, as a corollary of Theorem 1, in the case $t=1$. Bounds for $|x|$ and $|y|$ are also computed in [3].

For general $t$ we deduce, in [3], the following generalization of Runge's Theorem, as another corollary of Theorem 1.

THEOREM 2. Let $F(x, y)$ be irreducible in $\mathbf{Z}[x, y]$ and set

$$
F(x, y)=\sum_{i=1}^{d_{1}} x^{i} f_{i}(y)=\sum_{j=0}^{d_{2}} y^{j} g_{j}(x)
$$

where

$$
g_{d_{2}}(x)=a \prod_{r=1}^{v}\left(x-\alpha_{r}\right)^{\mu_{r}}, \quad f_{d_{1}}(y)=b \prod_{s=1}^{w}\left(y-\beta_{s}\right)^{\nu_{s}}
$$

and assume that there exist infinitely many L-lattice points $(x, y) \in \mathcal{O}_{L}^{2}$ which satisfy $F(x, y)=0$ and

$$
|1 / x| \leq c_{6}|x|^{1 /(t-1) d_{2}^{2}}, \quad|1 / y| \leq c_{6}|y|^{1 /(t-1) d_{1}^{2}},
$$

where $c_{6}$ is a positive constant. Assume one of the following conditions for these L-lattice points:

$$
\begin{array}{ll}
\left|1 /\left(x-\alpha_{r}\right)\right| \leq c_{7}|y|^{1 / \mu_{r}-c_{8}}, & r=1,2, \ldots, v, \\
\left|1 /\left(y-\beta_{s}\right)\right| \leq c_{7}|x|^{1 / \nu_{s}-c_{8}}, & s=1,2, \ldots, w,
\end{array}
$$

where $c_{7}, c_{8}$ are positive constants. Then $F(x, y)$ violates Runge's Condition. 
To see that Theorem 2 is a corollary of Theorem 1, we need to observe that $e \leq d_{2}$, with strict inequality if Runge's Condition holds.

2. Outline of the proof of Theorem 1. Let $\rho=1,2, \ldots, M$ and construct functions

$$
F(x, y ; \rho)=s^{\rho} \prod_{\sigma=1}^{s} \prod_{\epsilon=0}^{e-1}\left(y-\sum_{n=-m}^{N} a_{n}^{(\sigma)} \varsigma^{\epsilon} x^{-1 / e}\right)^{n} .
$$

Estimate the quantities $F\left(x^{(\tau)}, y^{(\tau)} ; \rho\right)$ to find, for $\tau=\tau_{0}$, that they are of the order of magnitude $O\left(|x|^{k}\right)$ where $k \leq M+(s e-1) m / e-c_{1}(N+1) / e$; whereas for a general $\tau$ they are $O\left(|x|^{c_{5} s N}\right)$. Observe that

$$
F(x, y ; \rho)=P(x, y ; \rho)+\sum_{\mu} \sum_{\nu} b_{\rho \mu \nu} y^{\nu} / x^{\mu}+O\left(x^{-1 / e}\right)
$$

where $P(x, y ; \rho)$ is a polynomial with rational coefficients, the $b_{\rho \mu \nu}$ 's are rational numbers, and where the double sum is extended over the $M$ values of $(\mu, \nu)$ indicated in the theorem. Construct integers $B_{\rho}$, not all zero, for which

$$
\sum_{\rho=0}^{M} B_{\rho} b_{\rho \mu \nu}=0
$$

for all $M$ pairs $(\mu, \nu)$, and for which

$$
Q(x, y)=\sum_{\rho=0}^{M} B_{\rho} P(x, y ; \rho) \in \mathbf{Z}[x, y] .
$$

From the estimations of $F\left(x^{(\tau)}, y^{(\tau)} ; \rho\right)$ we deduce that $Q(x, y)$ vanishes for all but a finite number of these $L$-lattice points $(x, y)$, and so there is a polynomial of the form

$$
P(x, y)=Q_{1}(x) Q(x, y) \in \mathbf{Z}[x, y],
$$

with $Q_{1}(x) \in \mathbf{Z}[x]$, that vanishes at all of these $L$-lattice points. Finally we argue that $\tilde{P}(x, y)$ and $P_{\lambda}(x, y)$ have the indicated properties.

Note that for the finiteness results of Theorems 1 and 2 over algebraic number fields some restrictions like (iv) and (v) in Theorem 2 are needed. For example the equation $(x-a)(y-b)=1$ satisfies Runge's Condition, but has infinitely many solutions, $x=a+\eta, y=b+\eta^{-1}$ in integers of a number field with infinitely many units $\eta$.

As a simple illustration we consider the problem: When is the product of four consecutive integers a square? That is, find the solutions of

$$
\begin{aligned}
y^{2} & =(x-1) x(x+1)(x+2)=x^{4}+2 x^{3}-x^{2}-2 x \\
& =\left(x^{2}+x-1\right)^{2}-1 .
\end{aligned}
$$

This equation satisfies Runge's Condition. In $\mathbf{Z}$ the only solutions are clearly $y=0, x=-2,-1,0,1$. It is equally easy to see that there are no other solutions in integers of imaginary quadratic fields, where the only new units to be considered are $\omega= \pm i,( \pm 1 \pm i \sqrt{3}) / 2$. Now equation (2.1) yields

$$
y+\left(x^{2}+x-1\right)=\omega, \quad y-\left(x^{2}+x-1\right)=-\bar{\omega}
$$


and hence

$$
y=\frac{1}{2}(\omega-\bar{\omega}), \quad x^{2}+x-1=\frac{1}{2}(\omega+\bar{\omega}) .
$$

For $\omega= \pm i$ the equation $x^{2}+x-1=0$ has no solution in the field and for $\omega=( \pm 1 \pm i \sqrt{3}) / 2$ the quantities $(\omega \pm \bar{\omega}) / 2$ are not integers.

In some real quadratic number fields there obviously are solutions with $y \neq 0$. Nevertheless the conditions of Theorem 2 are satisfied and we can get good bounds for the solutions. To see this let $\eta$ be the fundamental unit of the field. Then (2.1) yields

$$
y+\left(x^{2}+x-1\right)= \pm \eta^{n}, \quad y-\left(x^{2}+x-1\right)=\mp \eta^{-n},
$$

or

$$
y= \pm \frac{1}{2}\left(\eta^{n}-\eta^{-n}\right), \quad x^{2}+x-1= \pm \frac{1}{2}\left(\eta^{n}+\eta^{-n}\right) .
$$

So, if there are solutions for large $n$, then

$$
\left|y \sim \eta^{n} / 2, \quad\right| x \mid \sim\left(\eta^{n} / 2\right)^{1 / 2}
$$

while

$$
\left|1 / y \sim 2 / \eta^{n}, \quad\right| 1 / x \sim\left(\eta^{n} / 2\right)^{-1 / 2} .
$$

Thus condition (iv) of Theorem 2 is satisfied and condition (v) is vacuous. This yields the fact that the number of solutions is finite in integers of any real quadratic number field and the bounds can be readily computed, as we do in [3] for solutions in $\mathbf{Z}$.

The above argument is equally valid for all number fields with a cyclic group of units (other than the roots of unity), since in those cases $1 / x$ and $\mid 1 / y$ are small when $n$-and hence $|x|$ and $\mid y$-are large.

\section{REFERENCES}

1. D. L. Hilliker, An algorithm for solving a certain class of Diophantine equations. I, Math. Comp. 38 (1982), 611-626.

2. __ An algorithm for solving a certain class of Diophantine equations. II (to be submitted).

3. D. L. Hilliker and E. G. Straus, Determination of bounds for the solutions to those binary Diophantine equations that satisfy the hypotheses of Runge's theorem (to be submitted).

4. C. Runge, Ueber ganzzahlige Lösungen vor Gleichungen zwischen zwei Veränderlichen, J. Reine Angew. Math. 100 (1887), 425-435. See Jbuch. 19, 76-77.

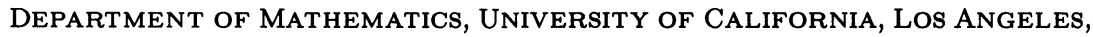
CALIFORNIA 90024 